\title{
Comunicación
}

\section{Frecuencia y clasificación de neoplasias orales en pacientes caninos de la Clínica de Animales Menores de la Universidad Nacional Mayor de San Marcos (2009-2013)}

\author{
Frequency and classification of oral neoplasms in canine patients at the Small
} Animal Clinic of the Universidad Nacional Mayor de San Marcos (2009-2013)

\author{
Rosalyn Hurtado Y. ${ }^{1}$, Viviana Fernández P.,3
}

\section{Resumen}

El presente estudio tuvo como objetivo determinar la frecuencia de neoplasias orales diagnosticadas histopatológicamente en caninos pacientes de la Clínica de Animales Menores de la Facultad de Medicina Veterinaria de la Universidad Nacional Mayor de San Marcos (Lima, Perú) en el periodo enero de 2009 a diciembre de 2013. Se consideraron las variables edad, sexo, peso, raza, localización anatómica y clasificación histológica. De 238 informes histopatológicos de pacientes caninos, 192 (80.7\%) fueron neoplasias y 23 $(12 \%)$ fueron neoplasias orales. Las neoplasias malignas fueron las de mayor presentación (73.9\%), siendo la de mayor frecuencia el melanoma oral (21.7\%), mientras que la neoplasia benigna más frecuente fue el épuli (17.4\%). Los machos fueron los más afectados $(65.2 \%)$. El grupo etario con mayor presentación de neoplasias fue entre 6 y 10 años $(52.2 \%)$ y de peso corporal entre 25 y $44 \mathrm{~kg}$ (47.8\%). Los caninos de razas definidas fueron los más afectados (69.6\%), especialmente el Rottweiler y el Labrador Retriever (13\% cada raza). La localización anatómica más común fue la gingiva (69.6\%).

Palabras clave: cavidad oral, neoplasia, canino

\section{ABSTRACT}

The aim of this study was to determine the frequency of oral neoplasms diagnosed histopathologically in canine patients of the Small Animal Clinic of the Faculty of Veterinary Medicine of the Universidad Nacional Mayor de San Marcos (Lima, Peru) in the period of

\footnotetext{
${ }^{1}$ Facultad de Medicina Veterinaria, Universidad Nacional Mayor de San Marcos, Lima, Perú

${ }^{2}$ Clínica de Animales Menores, Facultad de Medicina Veterinaria, Universidad Nacional Mayor de San Marcos, Lima, Perú

${ }^{3}$ E-mail:vfernandezp@unmsm.edu.pe

Recibido: 6 de septiembre de 2019

Aceptado para publicación: 6 de junio de 2020

Publicado: 11 de agosto de 2020
} 
January 2009 till December 2013. The variables age, sex, body weight, breed, anatomical location, and histological classification were considered. Of 238 histopathological reports of canine patients, $192(80.7 \%)$ were neoplasms and $23(12 \%)$ were oral neoplasms. Malignant neoplasms were of greater presentation $(73.9 \%)$, with oral melanoma being the most frequent (21.7\%), and the most frequent benign neoplasm was epulis (17.4\%). Males were mostly affected $(65.2 \%)$. The age group with the greatest presentation of malignancies was between 6 and 10 years $(52.2 \%)$ and the body weight was between 25 and $44 \mathrm{~kg}(47.8 \%)$. Purebred canines were the most affected (69.6\%), especially the Rottweiler and Labrador Retriever ( $13 \%$ each breed). The most common anatomical location was the gingiva $(69.6 \%)$.

Key words: oral cavity, neoplasms, canine

\section{INTRODUCCIÓN}

El avance de la Medicina Veterinaria ha logrado mejorar el control de las enfermedades infecciosas y disminuir la mortalidad de los animales de compañía, dado como resultado un notable incremento en la expectativa de vida de estos animales. Por lo tanto, las afecciones que se presentan en edades avanzadas, como las neoplásicas, vienen teniendo mayor relevancia, dado que es una de las principales causas de decesos. Se observa que entre el 15 y $20 \%$ de los canes adultos y gerontes atendidos en clínicas veterinarias padecen de procesos neoplásicos (Cuitiño, 2011).

La cavidad oral puede presentar una amplia gama de procesos patológicos que son de común hallazgo en la práctica clínica veterinaria. Entre las patologías de la cavidad oral se encuentran las neoplasias que son el cuarto cáncer más común en caninos (Nelson y Couto, 2010); las que pueden ser de origen odontogénico o no odontogénico, presentándose formas benignas y malignas.

El presente estudio muestra los resultados obtenidos de las historias clínicas durante los años 2009-2013 sobre las neoplasias en la cavidad de la oral de la Clínica de Animales Menores (CAME) de la Facultad de Medicina Veterinaria (FMV) de la Universidad Nacional Mayor de San Marcos (UNMSM) en Lima, Perú. La CAME es un centro con una gran variedad casuística y donde se cuenta con la especialidad de oncología veterinaria. El objetivo del estudio fue determinar la frecuencia de las neoplasias orales según las variables raza, sexo, edad, clasificación histológica y localización anatómica.

\section{Materiales y Métodos}

Se utilizaron los registros clínicos de la CAME-FMV-UNMSM del periodo enero de 2009 y diciembre de 2013. Se recuperaron datos de los protocolos de histopatología diagnosticados por el laboratorio de patología veterinaria de la FMV y otros. Los casos con resultado de neoplasias fueron divididos por su localización anatómica, utilizándose aquellos con resultado de neoplasia oral.

Se analizaron las variables de edad, raza, sexo, localización anatómica, diagnóstico y clasificación histológica. 
Cuadro 1. Frecuencia (\%) de neoplasias orales por grupo etario y peso corporal en pacientes caninos de la CAME-FMVUNMSM (2009-2013)

\begin{tabular}{ccc}
\hline Variable & & Porcentaje \\
\hline Grupo etario & $<1$ & 4.4 \\
(años) & $1-5$ & 21.7 \\
& $6-10$ & 52.2 \\
& $\geq 11$ & 21.7 \\
Peso & $<5$ & 8.7 \\
$(\mathrm{~kg})$ & $5-10$ & 8.7 \\
& $11-24$ & 30.4 \\
& $25-44$ & 47.8 \\
& $\geq 45$ & 4.4 \\
\hline Total & $(\mathrm{n}=23)$ & 100.0 \\
\hline
\end{tabular}

\section{Resultados}

El número de historias clínicas en el periodo en estudio fue de 23489 , de las cuales 19192 correspondieron a pacientes caninos, y de estas, 238 historias clínicas contaron con protocolos de histopatología.

En los protocolos histopatológicos en caninos se encontró una frecuencia de $80.7 \%$ (192/238) de lesiones neoplásicas y 19.3\% (46/238) de no neoplásicas. Las neoplasias orales en caninos representaron el 12.0\% (23/ 192). Por otro lado, de las 25 lesiones encontradas en la cavidad oral, 92\% (23/25) correspondieron a lesiones neoplásicas y el $8 \%$ $(2 / 25)$ a lesiones no neoplásicas.

Las frecuencias de neoplasias por grupo etario y peso corporal se presentan en el Cuadro 1, y por grado de malignidad, según sexo y raza en el Cuadro 2. La frecuencia de neoplasias orales por tipo histológico y localización anatómica se presemtan en el Cuadro 3.

\section{Discusión}

El $92 \%$ de las biopsias orales realizadas a pacientes caninos de la Clínica de Animales Menores de la FMV de la UNMSM fueron diagnosticadas como neoplasia oral. Frecuencia mayor al $37.8 \%$ reportado por Stenholt et al. (2009) del Registro Danés de Cáncer en Veterinaria de 60 centros veterinarios en Dinamarca. Esta diferencia podría deberse al tamaño muestral y a una mayor aceptación de los propietarios en los centros daneses a realizar biopsias orales,

El $12.0 \%$ de las neoplasias orales con relación al total de neoplasias diagnosticadas por histopatología fue igualmente mayor al $7.9 \%$ encontrado por Páucar et al. (2017) y De Sousa (2010), así como al 6\% reportado por Venturini (2006), posiblemente debido a que en la CAME se considera anestesiar a los pacientes para una mejor revisión de la cavidad oral, pudiendo de esa manera, de ser necesario, realizar una biopsia incisional o excisional de la lesión oral a fin de obtener un diagnóstico histopatológico. De no hacerse así, muchas veces se recurre a los diagnósticos citológicos de las neoplasias, los cuales no son diagnósticos definitivos.

La mayor frecuencia de neoplasias orales fue observada en caninos de 6 a 10 años (52.2\%), resultados que concuerdan con los reportes de Martin (2013) en 64 casos de neoplasias orales, quién encontró la mayor frecuencia (56.4\%) en caninos de 6-10 años, y por el de De Sousa (2010) con una mayor frecuencia en el rango de 7 a 13 años. Es de esperarse hallar una mayor frecuencia de neoplasias conforme avance la edad, dada una mayor activación de oncogenes y la presencia acumulada de elementos cancerígenos (Mendiz, 2004). 
Cuadro 2. Frecuencia de neoplasias orales por sexo y raza, según el nivel de malignidad en pacientes caninos de la Clínica de Animales Menores de la Facultad de Medicina Veterinaria, Universidad Nacional Mayor de San Marcos, Lima, Perú (2009-2013)

\begin{tabular}{llcccccc}
\hline \multirow{2}{*}{ Variable } & \multicolumn{2}{c}{ Maligno } & \multicolumn{2}{c}{ Benigno } & \multicolumn{2}{c}{ Total } \\
\cline { 2 - 7 } & & $\mathrm{n}$ & $\%$ & $\mathrm{n}$ & $\%$ & $\mathrm{n}$ & $\%$ \\
\hline Sexo & Macho & 12 & 52.2 & 3 & 13.0 & 15 & 65.2 \\
& Hembra & 5 & 21.7 & 3 & 13.0 & 8 & 34.8 \\
\cline { 2 - 7 } Raza & 4 & 17.4 & 3 & 13.0 & 7 & 30.4 \\
& Mestizo & 3 & 13.0 & 0 & 0 & 3 & 13.0 \\
& Rottweiler & 3 & 13.0 & 0 & 0 & 3 & 13.0 \\
& Labrador Retriever & 2 & 8.7 & 0 & 0 & 2 & 8.7 \\
& Pastor Alemán & 1 & 4.4 & 1 & 4.4 & 2 & 8.7 \\
& Shih Tzu & 0 & 0 & 1 & 4.4 & 1 & 4.4 \\
& Cocker Spaniel & 1 & 4.4 & 0 & 0 & 1 & 4.4 \\
& Dogo Argentino & 0 & 0 & 1 & 4.4 & 1 & 4.4 \\
Golden Retriever & 1 & 4.4 & 0 & 0 & 1 & 4.4 \\
Bóxer & 1 & 4.4 & 0 & 0 & 1 & 4.4 \\
Schnauzer & 1 & 4.4 & 0 & 0 & 1 & 4.4 \\
Pekinés & 17 & 73.9 & 6 & 26.1 & 23 & 100.0 \\
\hline
\end{tabular}

La frecuencia de neoplasias orales en machos fue de $65.2 \%$, mientras que en hembras fue de $34.8 \%$. En este sentido, Fonseca et al. (2011) encontraron frecuencias de 53.3 y $46.7 \%$ para machos y hembras, respectivamente. Asimismo, De Sousa (2010) indica que, en Brasil, los machos tienen 2.4 veces mayor riesgo de desarrollar este tipo de cáncer que las hembras. No obstante, no se encontraron reportes que plantearan influencias hormonales, por lo que no se reporta predisposición sexual en la literatura para el cáncer oral (Fonseca et al., 2011; Mendiz, 2004).

En el presente estudio se encontró la mayor frecuencia de neoplasias orales en perros con pesos de $25-44 \mathrm{~kg}$ (47.8\%), especialmente por presentación de melanoma y rabdomiosarcoma, seguidos de perros con pesos entre 11 y $24 \mathrm{~kg}$ con un $30.4 \%$. Asimismo, caninos de 18 y $27 \mathrm{~kg}$ presentaron carcinoma de células escamosas y un canino de $40 \mathrm{~kg}$ presentó fibrosarcoma oral, lo cual corrobora que los perros de razas grandes presentan mayores probabilidades de adquirir carcinoma de células escamosas y fibrosarcomas (Howard, 2000; Gioso, 2003). Por otro lado, Birchard (1996) reporta una mayor incidencia de fibrosarcoma oral en perros grandes con pesos mayores de $23 \mathrm{~kg}$.

Las razas definidas como grupo fueron las más afectadas (69.7\%) en comparación con los perros mestizos (30.3\%). Páucar et al. (2017), también reporta una frecuencia de $75.2 \%$ para el grupo de razas definidas. 
Cuadro 3. Frecuencia de neoplasias orales por tipo histológico y localización anatómica en pacientes caninos de la Clínica de Animales Menores de la Facultad de Medicina Veterinaria, Universidad Nacional Mayor de San Marcos, Lima, Perú (2009-2013)

\begin{tabular}{|c|c|c|c|c|c|c|c|c|c|c|}
\hline \multirow{3}{*}{ Neoplasias orales } & \multicolumn{8}{|c|}{ Localización anatómica } & \multirow{2}{*}{\multicolumn{2}{|c|}{ Total }} \\
\hline & \multicolumn{2}{|c|}{ Gingiva } & \multicolumn{2}{|c|}{ Labio } & \multicolumn{2}{|c|}{ Paladar } & \multicolumn{2}{|c|}{ Lengua } & & \\
\hline & $\mathrm{n}$ & $\%$ & $\mathrm{n}$ & $\%$ & $\mathrm{n}$ & $\%$ & $\mathrm{n}$ & $\%$ & $\mathrm{n}$ & $\%$ \\
\hline Melanoma & 3 & 13.0 & 1 & 4.4 & 1 & 4.4 & 0 & 0 & 5 & 21.7 \\
\hline Épuli & 4 & 17.4 & 0 & 0 & 0 & 0 & 0 & 0 & 4 & 17.4 \\
\hline Rabdomiosarcoma & 2 & 8.7 & 0 & 0 & 0 & 0 & 1 & 4.4 & 3 & 13.0 \\
\hline Plasmocitoma & 2 & 8.7 & 1 & 4.4 & 0 & 0 & 0 & 0 & 3 & 13.0 \\
\hline Papiloma & 1 & 4.4 & 1 & 4.4 & 0 & 0 & 0 & 0 & 2 & 8.7 \\
\hline $\begin{array}{l}\text { Carcinoma de } \\
\text { células escamosas }\end{array}$ & 1 & 4.4 & 0 & 0 & 1 & 4.4 & 0 & 0 & 2 & 8.7 \\
\hline Fibrosarcoma & 1 & 4.4 & 0 & 0 & 0 & 0 & 0 & 0 & 1 & 4.4 \\
\hline $\begin{array}{l}\text { Sarcoma } \\
\text { indiferenciado }\end{array}$ & 1 & 4.4 & 0 & 0 & 0 & 0 & 0 & 0 & 1 & 4.4 \\
\hline Hemangiosarcoma & 1 & 4.4 & 0 & 0 & 0 & 0 & 0 & 0 & 1 & 4.4 \\
\hline Linfoma & 0 & 0 & 1 & 4.4 & 0 & 0 & 0 & 0 & 1 & 4.4 \\
\hline Total & 16 & 69.6 & 4 & 17.4 & 2 & 8.7 & 1 & 4.4 & 23 & 100.0 \\
\hline
\end{tabular}

Las frecuencias de neoplasias orales por raza dependen mayormente de la frecuencia de determinadas razas según la zona geográfica y el estrato socioeconómico. Así por ejemplo, en Brasil, Venturini (2006) encontró una mayor frecuencia en Bóxer en la ciudad de en São Paulo, Gomes et al. (2009) en Porto Alegre encontraron al Poodle, y Fonseca et al. (2011) en Brasilia encontró al Doberman Pinscher como la raza mayor frecuencia de neoplasias orales.

Las neoplasias no odontogénicas en este estudio representaron el $82.6 \%$, similar resultado a lo descrito por Páucar et al. (2017) con $80 \%$. Esto se debería a que las neoplasias odontogénicas se forman solo a partir de tejido que participa en la odonto-génesis, por lo que son raras en todas las especies.
Las neoplasias orales malignas representan el $73.9 \%$, lo que coincide con el $72 \%$ obtenido por Páucar et al. (2017), pero superior a lo encontrado en otros reportes (Venturini, 2006; Stenholt et al., 2009). Por otro lado, las neoplasias orales benignas representaron el $26.1 \%$, valor menor lo presentado en otros reportes (Venturini, 2006; Stenholt et al., 2009; De Sousa, 2010), lo cual podría deberse a la baja frecuencia de caninos menores de un 1 año en este estudio, toda vez que la frecuencia de las neoplasias malignas aumenta con la edad. La papilomatosis oral es la neoplasia oral benigna más común en caninos jóvenes e inmunológicamente comprometidos (Lucena et al., 1996; Liptak y Withrow, 2013). 
La neoplasia maligna con más frecuencia fue el melanoma (21.7\%), posiblemente debido a que varias razas descritas en este estudio tienen la mucosa oral pigmentada, por lo que presentarían mayor riesgo a desarrollar este tipo de neoplasia (Ramos-Vara et al., 2000). La segunda neoplasia maligna más frecuente fue tanto el plasmocitoma como el rabdomiosarcoma (13.0\%), mientras que autores como Venturini (2006) reportó 9.9\% de frecuencia para rabdomiosarcoma oral y Páucar et al. (2017) encontraron el 26\%.

La mayor frecuencia de neoplasias orales benignas fue el épuli (17.39\%), seguido del papiloma (8.7\%). El épulis fibromatoso fue más frecuente que el épuli osteomatoso, lo cual coincide con Páucar et al. (2017) que obtuvo una frecuencia de $56 \%$ en épuli fibromatoso. Cabe resaltar que Liptak y Withrow (2013) clasifican al épuli osificante y épuli fibromatoso como fibroma odontogénico periférico.

En cuanto a las neoplasias dentro de la cavidad oral, el componente oral más afectado fue la gingiva $(69.7 \%)$, seguido del labio (17.4\%) y el paladar (8.7\%), lo cual presenta valores relativamente similares con otros reportes (Birchard, 1996; Mendiz, 2004; Páucar et al., 2017).

\section{Conclusiones}

- La frecuencia de neoplasias orales en caninos fue de $11.98 \%$ para el periodo 2009-2013.

- Las razas definidas fueron las más afectadas con $69.6 \%$, teniendo una mayor presentación el Rottweiler y el Labrador Retriever (13.0\% por raza).

- Las neoplasias orales más comunes son las no ontogénicas (82.6\%).

- Las neoplasias orales malignas fueron más frecuentes $(73.9 \%)$, siendo el melanoma el de mayor presentación $(21.7 \%)$.
- La localización anatómica más afectada fue la gingiva (69.7\%).

\section{Literatura Citada}

1. Birchard S. 1996. Surgical management of neoplasms of the oral cavity in dogs and cats. In: $20^{\text {th }}$ Annual Waltham/OSU Symposium for the Treatment of Small Animal Diseases: Oncology and Hematology. USA.

2. Cuitiños M. 2011. Neoplasias melanocíticas en caninos: evaluación de marcadores inmunohistoquímicos que contribuyen a la formulación del diagnóstico del pronóstico. Tesis Doctoral. Argentina: Univ. Nacional de la Plata. 200 p.

3. De Sousa I. 2010. Estudio retrospective das neoplasias em canídeos e felídeos domésticos, analisadas pelo Laboratorio de Anatomía Patológica da Faculdade de Medicina Veterinária da Universidade Técnica de Lisboa, no período compreendido entre 2000 e 2009. Tese de Mesgtrado. Lisboa: Universidade Técnica de Lisboa. 97 p.

4. Fonseca C, Goncalves A, Eidi L, Alves $R$, Dos Santos S. 2011. Estudo retrospectivo das neoplasias em cavidade oral atendidas no serviço de clínica médica do Hospital Veterinário da Faculdade UPIS, Brasilia-DF. Vet Zootec 18: 40-43.

5. Gioso MA. 2003. Neoplasia de cavidade oral. En: Odontología para o clinico de pequenos animais. $5^{\text {a }}$ ed. Sao Paulo: Minha Ed. p 133-152.

6. Gomes C, Oliveira L. Elizeire M, De Oliveira M, Oliveira R. Contesini E. 2009. Avaliação epidemiológica de cães com neoplasias orais atendidos no Hospital de Clínicas Veterinárias da Universidade Federal do Rio Grande do Sul. Ciênc Anim Bras 10: 835-839.

7. Howard P. 2000. Neoplasm of the maxilla and mandibula. In: Birchard SJ, Sherding RG (eds). Manual of small animal practice. $2^{\text {nd }}$ ed. Philadelphia: Saunders. p 1076-1078. 
8. Liptak J, Withrow S. 2013. Small animal clinical oncology. $5^{\text {th }}$ ed. Missouri: Elsevier Saunders. p 381-398.

9. Lucena R, Ginel P, Pérez J, López, R, Chacón F. Mozos E. 1996. Tumores de cavidad oral en el perro: estudio retrospectivo. AVEPA 16: 71-76.

10. Martin E. 2013. Frequence relative des tumeurs buccales chez le chien: étude retrospective de 62 cas. These de Docteur Vétérinaire. Lyon: Université Claude Bernard-Lyon I. $116 \mathrm{p}$.

11. Mendiz D. 2004. Descripción clínica y epidemiológica de neoplasias orales en caninos y felinos. Tesis de Médico Veterinario. Santiago: Universidad de Chile. $53 \mathrm{p}$.

12. Nelson R, Couto C. 2010. Medicina interna de pequeños animales. $4^{\mathrm{a}}$ ed. Madrid: Elsevier. $1504 \mathrm{p}$.

13. Páucar J, Perales $R$, Tabacchi $l$. 2017. Frecuencia de neoplasias en cavidad oral de caninos y felinos, diagnosticadas histopatológicamente en el Laboratorio de Patología Veterinaria de la
Universidad Nacional Mayor de San Marcos (periodo 2007- 2013). Rev Inv Vet Perú 28: 201-206. doi: 10.15381/ rivep.v28i1.12929

14. Ramos-Vara JA, Beissenherz ME, Miller MA, Johnson GC, Pace LW, Fard A, Kottler SJ. 2000. Retrospective study of 338 canine oral melanomas with clinical, histologic, and inmunohistochemical review of 129 cases. Vet Pathol 37: 597-608. doi: 10.1354/vp.37-6-597

15. Stenholt G, Bjorn L, Kristensen A, Kortegaard H, Eriksen T. 2009. Diagnostiske aspekter af oral onkologi hos hund og hat: Et retrospektivt studie af orale biopsier og sammenligning med tilfaelde af oral neoplasi rapporteret til Dansk Veterinaer Cancerregister. Dansk Veterinaertidsskrift 7: 20-22.

16. Venturini M. 2006. Estudo retrospectivo de 3055 animais atendidos no Odontovet (Centro Odontológico Veterinário) durante 44 meses. Tese de Mestrado. São Paulo: Universidade de São Paulo. 103 p. 